\title{
Métodos Prospectivos Implementados en la Vigilancia Tecnológica y Prospectiva de Vehículos Eléctricos (EV) y Tecnologías Periféricas en Colombia*
}

\section{Prospective Methods Implemented During the Development of Technology Forecasting of Electrical Vehicles (EVs) and Prospective Technologies in Colombia}

\author{
DOI: http://dx.doi.org/10.17981/ingecuc.11.2.2015.06
}

Articulo de investigación - Fecha de Recepción: febrero 27 de 2015 - Fecha de Aceptación: septiembre 15 de 2015

María Victoria Díaz Merchán

Magíster en Ingeniería Eléctrica. Electrical Machines \& Drives - Research Group., Universidad Nacional de Colombia. Bogotá (Colombia).mvdiazm@unal.edu.co

Javier Rosero García

Doctor en Ingeniería Electrónica. Electrical Machines \& Drives - Research Group., Universidad Nacional de Colombia. Bogotá (Colombia). jaroserog@unal.edu.co

Omar Fredy Prías Caicedo

Magíster en Eficiencia Energética. GRISEC., Universidad Nacional de Colombia. Bogotá (Colombia). ofpriasc@unal.edu.co

Para citar este artículo:

M. V. Díaz Merchán, J. Rosero García and O. F. Prías Caicedo, "Métodos Prospectivos Implementados en la Vigilancia Tecnológica y Prospectiva de Vehículos Eléctricos (EV) y Tecnologías Periféricas en Colombia,” INGE CUC, vol. 11, no. 2, pp. 59-67, 2015. DOI: http://dx.doi.org/10.17981/ingecuc.11.2.2015.06

\begin{abstract}
Resumen-En este artículo se presentan los métodos prospectivos implementados durante el desarrollo de la vigilancia tecnológica en vehículos eléctricos y tecnologías periféricas en Colombia. Estos métodos se desarrollan con el propósito de identificar las líneas de investigación relacionadas y las tecnologías emergentes que propendan por la innovación. Las metodologías aplicadas fueron las rondas Delphi y el planteamiento de escenarios futuros; ambas metodologías se implementaron como herramientas para identificar los principales temas de investigación que giran alrededor de los EV en Colombia. Los temas seleccionados, posterior al desarrollo de la vigilancia tecnológica, fueron debatidos por un grupo de expertos que contextualizaron la información obtenida para Colombia. A partir de esto se construyeron los escenarios de análisis. Se determinaron las tecnologías que pueden obstaculizar la incursión de los EV en Colombia, así como las tecnologías con mayor tendencia al consumo y los aspectos regulatorios más influyentes.
\end{abstract}

Palabras claves - Vigilancia Tecnológica, Prospectiva, Vehículo Eléctrico, Rondas Delphi, Planteamiento de Escenarios

\begin{abstract}
This paper presents prospective methods implemented during the development of Surveillance Technology in Electrical Vehicles and Prospective Technologies in Colombia. These methods are developed for identifying related lines of research and emerging technologies which foster innovation. Methodologies are Delphi rounds and the planning of future scenarios. Both methodologies are implemented as tools to identify research topics that revolve around EVs in Colombia. The topics selected following the development of technology forecasting, were discussed by a group of experts who contextualized the information obtained from Colombia. From this analysis, scenarios were constructed. Technologies hampering the incursion of EVs in Colombia were determined, as well as the technologies with the greatest trend to be consumed and the most influential regulatory aspects.
\end{abstract}

Keywords - Technology Forecasting, Prospective, Electrical Vehicles, Delphi Rounds, Planning of Scenarios

\footnotetext{
"Artículo de investigación científica derivado del proyecto de investigación titulado: "Evaluación de desempeño tecnológico y condiciones de operación de Vehículos Eléctricos (EV) y sistemas asociados para su funcionamiento en Colombia. EDCO-EVs". Financiado por Colciencias y Codensa SA-ESP. Fecha de inicio: enero de 2012. Fecha de finalización: diciembre de 2014.
} 


\section{INTRODUCCION}

Las instituciones educativas y el sector empresarial colombiano tienen la necesidad de conocer las más recientes tecnologías disponibles y los últimos desarrollos relacionados con los EV que se encuentran en el mercado. Esto hace importante conocer las líneas de investigación, las tecnologías emergentes y las tecnologías potencialmente obsoletas. Con ese objetivo, se realizan estudios de vigilancia tecnológica (VT), que mediante una metodología previamente establecida, detecta fuentes de información que permitan enfrentar decisiones de tipo tecnológico. De esta manera, es posible extraer información relevante sobre tendencias, novedades, invenciones, aplicaciones, tecnologías emergentes, y demás; simultáneamente, permite contemplar aspectos regulatorios y de mercado que pueden condicionar el éxito de una innovación tecnológica [1], [2] y [3].

Anticiparse a los cambios y a sus efectos aventaja de manera considerable a fabricantes y comercializadores de productos. Asimismo, la detección temprana de novedades y la percepción oportuna de amenazas, permite desarrollar de forma rápida y versátil estrategias para adaptarse a las tendencias. Con base en esta información, las empresas pueden reorientar sus recursos, esfuerzos y capacidades tecnológicas, productivas y comerciales. Por otro lado, estos ejercicios permiten que las entidades educativas fortalezcan las líneas de investigación para optimizar procesos y productos. Considerando lo anterior, se desarrollan procesos prospectivos mediante metodologías de vigilancia tecnológica conocidos como Rondas Delphi.

La metodología para el desarrollo de la prospectiva tecnológica permite encaminar los desarrollos en un período de tiempo para un fin determinado. Esta metodología está siendo promovida en Colombia desde el año 2004 con el fin de elaborar escenarios a futuro en sectores estratégicos, centrando esfuerzos en la investigación de nuevas tendencias y otras que pudieran surgir de la combinación de factores sociales, políticos nacionales y descubrimientos científicos.

Las rondas Delphi hacen parte de los ejercicios de prospectiva tecnológica y se desarrollan en fases consecutivas. Dentro de cada ronda se presentan contradicciones o divergencias que son transformadas en preguntas concretas para poder ser validadas por expertos en el tema; esto permite contextualizar la información adquirida. En el caso de los EV en Colombia, la información recopilada es obtenida principalmente de otros países con características regionales diferentes. Las rondas realizadas se centraron en la discusión de los temas de mayor relevancia; esto se tomó como base para el desarrollo del planteamiento de escenarios.
Para este ejercicio de prospectiva tecnológica de carácter académico, las características que se tuvieron en cuenta fueron las asociadas al tema tecnológico, temas ambientales y de regulación. El ejercicio integró los temas relacionados con el sector público, empresarial y los intereses de cada sector en relación con los productos.

El desarrollo de los procesos metodológicos de vigilancia tecnológica y prospectiva de carácter académico, así como las principales conclusiones y temas relevantes que surgieron de la implementación, se presentan en las siguientes secciones.

\section{Marco Teórico}

La prospectiva tecnológica (PT) se centra en la investigación de nuevas tendencias y otras que pudieran surgir de la combinación de factores sociales, políticos nacionales y descubrimientos científicos [4],[5],[6],[7] y [8].

Las técnicas específicas de prospectiva se dividen principalmente en dos categorías: las exploratorias y las normativas. Las primeras se centran en el análisis comparativo dentro de un marco temporal de datos históricos, parámetros técnicos y el resultado económico. Este análisis permite generar curvas o modelos a partir de esta información.

Las técnicas normativas empiezan proponiendo el estado deseado y trabajan de manera contraria a las exploratorias para determinar los pasos necesarios y, de este modo, conseguir el resultado requerido. El número de posibilidades de desarrollo desde la posición actual hasta el estado deseado pueden variar desde "ninguno" (tecnología totalmente nueva), hasta "varios". Cada posibilidad se analiza por su relevancia y dificultad.

Los métodos para hacer este tipo de pronósticos se pueden resumir en tres: los extrapolativos, basados en la extrapolación de variables medidas cuantitativamente que se encuentran disponibles en series históricas relativamente largas y consistentes; los métodos explicativos, que intentan construir modelos analíticos que vinculen variables dependientes e independientes a partir del establecimiento de relaciones de incidencia; y los métodos llamados subjetivos, que recogen la opinión de personas especialmente elegidas en función de su experiencia, habilidades o conocimientos en el tema de interés.

Las rondas Delphi corresponden a métodos subjetivos, y fueron desarrolladas por Rand Corporation aproximadamente hacia el año 1964 [9],[10],[11] y [12].

\section{A. Rondas Delphi}

Este método se constituye en una de las formas más aceptadas para la realización de prospectiva 
tecnológica. Se basa en la consulta a una serie de expertos que expresan sus opiniones en torno a un tema. La opinión, anónima en la primera ronda, es dada a partir de sus conocimientos y conceptos.

Los expertos e instituciones seleccionadas abarcan áreas como política, economía, social, cultural, ambiental, tecnológica y organizacional (PESCATO). Estos deben ser capaces de emitir un juicio sustentado acerca de la temática seleccionada, en este caso los EV en Colombia.

El proceso repetitivo de las rondas Delphi se basa en la elaboración de cuestionarios dirigidos a expertos. Cada vez que se recibe la respuesta de este grupo selecto, se procesa la información y se vuelve a estructurar otro cuestionario enfocado a las preguntas inconclusas o que muestran divergencia entre los resultados [4]. Estos planteamientos deben retroalimentar el diseño inicial de la encuesta que se realizó teniendo en cuenta los resultados de la etapa de vigilancia tecnológica y las temáticas de interés para el estudio.

Los principales pasos para la ejecución se describen a continuación [4]:

1. Formulación del problema o problemática a tratar

2. Elección de expertos que abarquen el PESCATO

3. Elaboración y divulgación de cuestionarios

4. Desarrollo, análisis y explotación de resultados

La metodología de las rondas Delphi se resume en la Fig. 1.

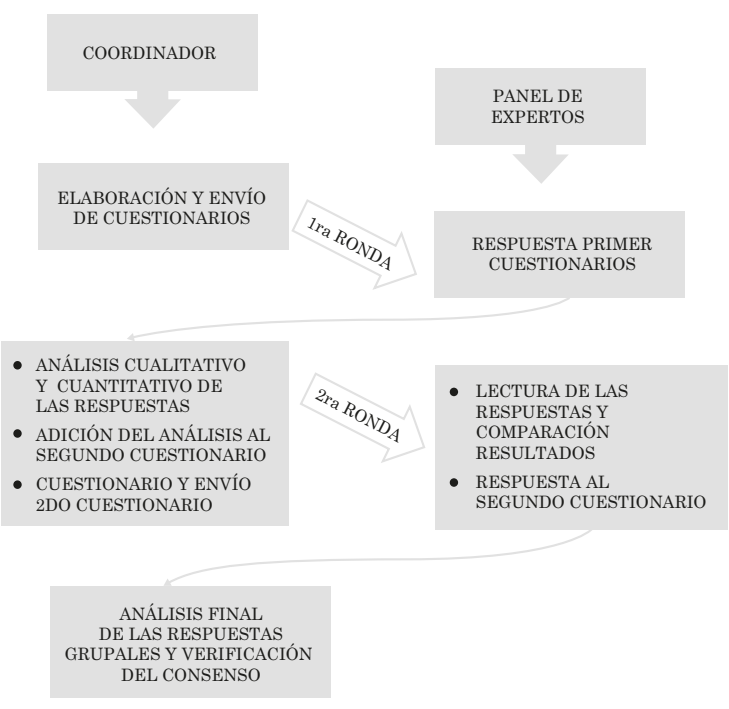

Fig. 1. Esquema general de un estudio de PT con el método Delphi. Fuente: [13]

\section{B. Planteamiento de Escenarios}

La construcción de escenarios no implica anticipar el futuro, porque no existen estadísticas sobre tiempos no ocurridos. Los escenarios permiten re- ducir las incertidumbres que se pueden presentar. El propósito del establecimiento de escenarios no es saber lo que va a suceder, sino saber qué diferencias habrá en la ocurrencia de las tecnologías, o la forma en que éstas se presentarán [4], [7].

Un escenario puede definir cómo modelar una situación futura esperada [5]. Existen tres tipos de escenarios de acuerdo con su descripción:

- Tendenciales: Corresponde a los escenarios que tienden a acontecer mayormente. Se obtienen de la evolución futura con base en proyecciones de tendencias históricas [7], [10].

- Normativos (optimistas, pesimistas): Estos escenarios son los que deben de acontecer. Corresponden a las potencialidades deseables [7], [10].

- Exploratorios: Son escenarios que pueden acontecer. Posibilidad de futuros alternativos [7], [10].

La metodología para el desarrollo de los posibles escenarios se describe en los siguientes pasos [8] [12].

1. Identificación de la situación o diagnóstico problematizado

2. Descripción de la trayectoria futura más estimable y las posibles acciones

3. Aproximación de las situaciones o riesgos de azar que pueden manifestarse

4. Definición del horizonte de tiempo

5. Descripción de la situación futura

\section{III.Rondas Delphi en los EV y Resultados}

De los resultados obtenidos con la vigilancia tecnológica se derivaron las primeras preguntas para desarrollar en las rondas Delphi. Los principales temas fueron:

- Aplicaciones de los EV

- Tipos y centros de recarga

- Tecnologías periféricas

Los principales resultados de cada una de las rondas son expuestos a continuación [13].

\section{A. Primera Ronda Delphi}

Esta encuesta consideró los siguientes aspectos en las preguntas para el grupo de expertos de 17 profesiones [13]:

- Tipo de aplicación o uso de tipos de los EV por segmentos (Fig. 2)

- Características de las baterías para los EV (Fig. 3)

- Tecnologías periféricas de los EV ( Fig. 4)

- Supercondensadores como alternativa para el almacenamiento de energía (Fig. 5)

- Problemática de las baterías actuales (Fig. 6)

- Tipo de recarga para los EV (Fig. 7)

- Tipos de centro de carga de cada uno de los usos (Fig. 8)

- Impactos de la implementación de los EV (Fig. 9) 


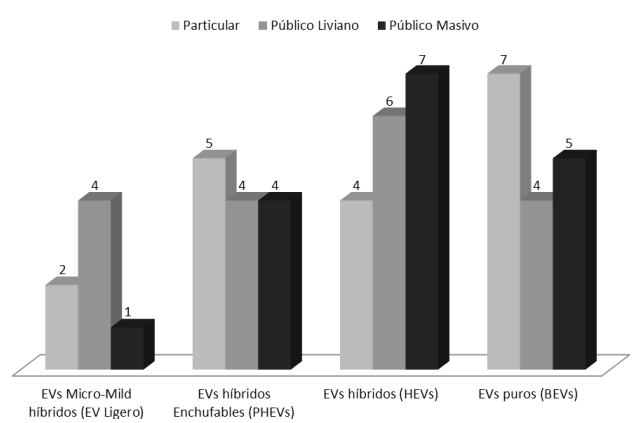

Fig. 2. Resultados Primera Ronda Delphi Tipo de Aplicaciones para los EV.

$$
\text { Fuente: [13] }
$$

\section{Características de las Baterías}

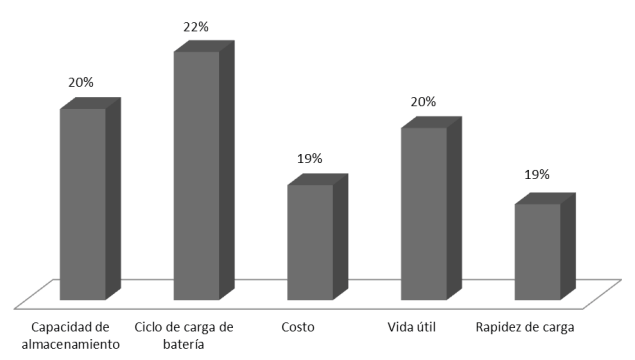

Fig. 3. Resultados Primera Ronda Delphi Características de las Baterías para los EV. Fuente: [13]

\section{Desarrollos de Tecnologías}

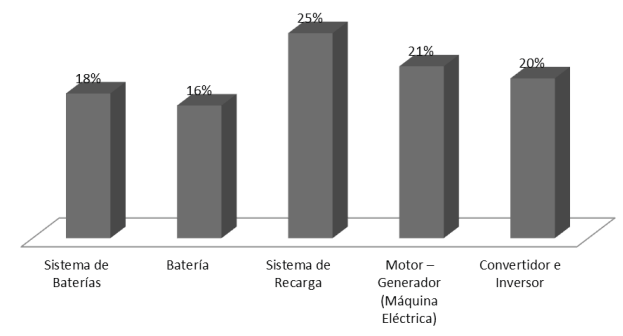

Fig. 4. Resultados Primera Ronda Delphi Tecnologías Periféricas de los EV. Fuente: [13]

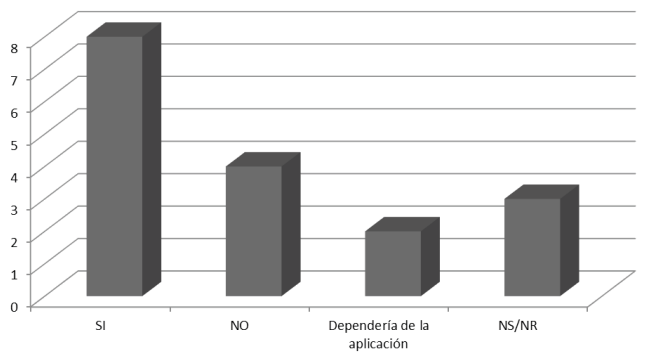

Fig. 5. Resultados Primera Ronda Delphi Supercondensadores como alternativa de almacenamiento. Fuente: [13]

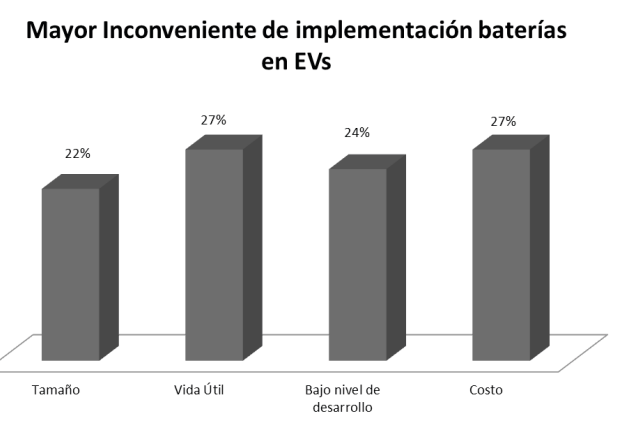

Fig. 6. Resultados Primera Roda Delphi - Principal Obstáculo en el Desarrollo de las Baterías. Fuente: [13]

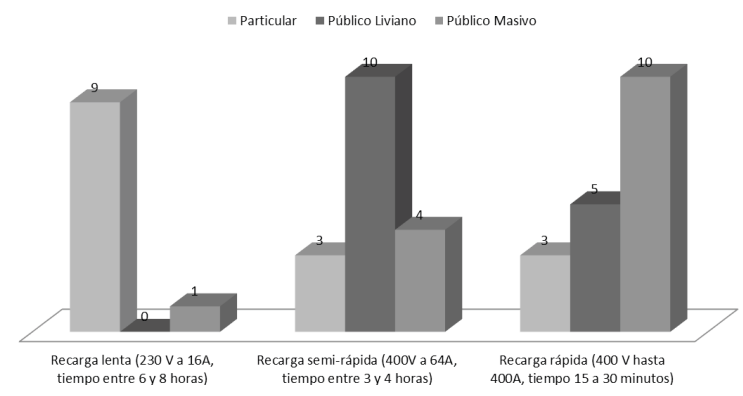

Fig. 7. Resultados Primera Ronda Delphi Tipo de Recarga para Cada Tipo de Uso. Fuente: [13]

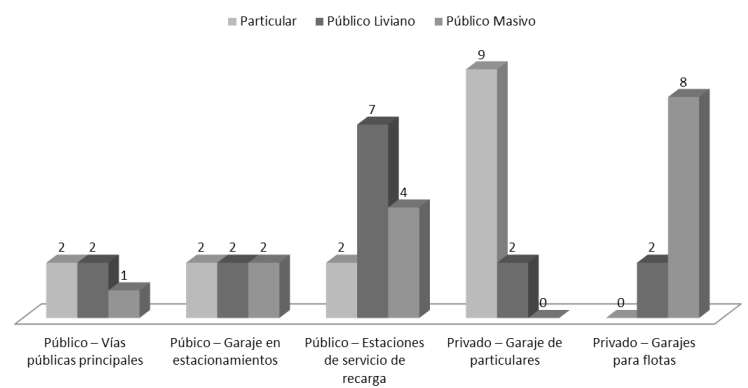

Fig. 8. Resultados Primera Ronda Delphi Centro de Recarga para Cada Tipo de Uso.

$$
\text { Fuente: [13] }
$$

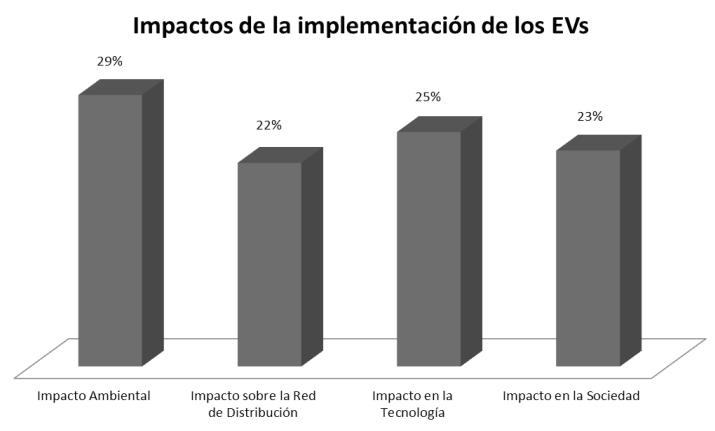

Fig. 9. Resultados Primera Ronda Delphi Impacto sobre la Implementación de los EV. Fuente: [13] 
Los resultados de la primera ronda Delphi, de acuerdo con las respuestas de los expertos, son los siguientes:

\section{1) Tecnologías Aplicables en Colombia de Acuerdo con el Uso:}

- La tecnología recomendada para los vehículos de transporte público masivo es: Vehículo Eléctrico Hibrido (HEV) y Vehículo Eléctrico de Batería (BEV).

- Para el caso de transporte público liviano y particular liviano no se cuenta con tecnología definida.

\section{2) Tecnologías Periféricas:}

- No se presenta claridad en cuanto a la tecnología de mayor desarrollo alrededor de los EV.

- Los supercondensadores se presentan como tecnología emergente.

\section{3) Tipos y Centros de Recarga:}

- La recarga lenta (tiempo entre 6 y 8 horas) es la mejor opción para vehículos particulares.

- Para vehículos de uso público liviano se resalta la opción de recarga semi - rápida (tiempo entre 3 y 4 horas).

- La instalación de centros de recarga en garajes privados se haría para para vehículos particulares.

- Para vehículos livianos de uso público se resalta la opción de estaciones de servicio de recarga pública (vías).

- La recarga para vehículos de uso público masivo se considera en los garajes de flotas privadas

\section{B. Segunda Ronda Delphi}

Luego del procesamiento de los resultados de la primera encuesta, se estableció un nuevo cuestionario con preguntas que no mostraron unanimidad [13]. Esta encuesta ofreció dos opciones de respuesta y, adicionalmente, opción de explicación de la respuesta. El propósito era confirmar los resultados de la encuesta anterior y encontrar unanimidad de resultados en los siguientes aspectos:

- Pregunta 1: ¿Son los BEV adecuados para uso particular urbano?

- Pregunta 2: ¿Para el año 2020 se tendría un 20\% de estos vehículos en el mercado?

- Pregunta 3. ¿Son los HEV adecuados para uso en transporte liviano masivo?

- Pregunta 4. ¿Debe el gobierno nacional o local establecer normatividades e incentivos para generar mercado de los EV en Colombia?

- Pregunta 6: ¿El impacto ambiental en la disminución del material particulado se compensa con el cambio del parque automotor y la disposición final de las baterías de los EV?
- Pregunta 7: ¿Es adecuado instalar estaciones de recarga semi-rápida e infraestructura pública para el transporte público liviano?

El resultado estadístico que se obtuvo se muestra en la Fig. 11. Estos resultados fueron argumentados por cada uno de los actores de acuerdo con su experiencia y sus investigaciones. Los resultados de la segunda ronda Delphi son:

\section{1) Tecnologías Aplicables en Colombia de Acuerdo con el Uso:}

- Se reafirma que la tecnología para vehículos livianos de uso particular apropiada es el BEV, recomendando puntualmente el city-car.

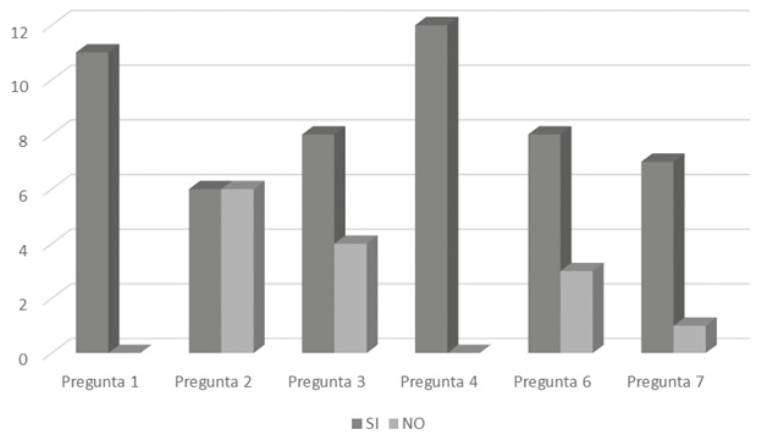

Fig. 10. Resultados Segunda Ronda Delphi. Fuente: [13]

- Se debe optimizar el costo, tamaño y autonomía de las baterías.

- Se recomienda la tecnología HEV para la implementación al inicio del plan de masificación como vehículos livianos y masivos de uso público.

\section{2) Tecnologías Periféricas:}

- Se reafirma la carencia de desarrollo tecnológico local en cada una de las tecnologías periféricas planteadas.

- La batería es el sistema de almacenamiento más representativo. Su capacidad de almacenamiento y ciclo de carga son características importantes, además de su seguridad, costo, tamaño y ubicación en el vehículo, vida útil, autonomía y disposición final.

\section{3) Tipos y Centros de Recarga}

- Las infraestructuras de recarga para vehículos de servicio público, dependerán en gran parte, de los gobiernos locales.

- En general, el sistema de recarga debe ser rápido, independiente de si es masivo o liviano, pues el exceso de tiempo implicaría pérdida de dinero.

- Se deben unificar los conectores de recarga sobre todo para vehículos particulares. 


\section{4) Impactos}

- La reducción del material particulado y el ruido en el ambiente se obtendría con la implementación masiva de los EV.

- La educación es uno de los factores más importantes en cuanto a los impactos; se deben inculcar nuevos conceptos encaminados al uso de tecnología asociada a los EV.

- Se plantea estudiar la disposición de uso final de los elementos de los vehículos de combustión versus la penetración de los EV para evaluar el beneficio ambiental de la implementación.

En la segunda ronda Delphi se destacó que el gobierno debe establecer incentivos y normatividad tanto ambiental como energética adecuada dentro de un marco global, teniendo en cuenta las políticas del exterior y contextualizándolas con la realidad colombiana junto con una estrategia de cambio cultural.

\section{Tercera Ronda Delphi- Taller de Expertos}

La tercera ronda Delphi se realizó por medio de un taller en el que se socializó la investigación de vigilancia tecnológica y se realizó un debate con base en las investigaciones socializadas y en premisas alrededor de los EV. Este ejercicio sirvió para conocer las opiniones de los participantes y que éstos a su vez conocieran las opiniones de los otros.

Las principales conclusiones y comentarios se resumen y clasifican a continuación:

\section{1) Incorporación de Nuevas Tecnologías en un País como Colombia}

- La cultura colombiana y los acuerdos internacionales actuales son dos aspectos que deben tenerse en cuenta para que Colombia vaya a la vanguardia de los desarrollos tecnológicos.

- La red de distribución de energía debe ser evaluada y reconfigurada para garantizar su capacidad de suministro frente a la masificación de los EV.

- Colombia tiene mayor capacidad de adaptación a la tecnología que a la investigación y al desarrollo para la masificación de los EV [13].

\section{2) Sistemas de Recarga}

- La implementación de los sistemas de recarga para los EV en Colombia se va a iniciar en el sector público.

- La infraestructura adecuada en sistemas de recarga generará confiabilidad para la masificación de los EV.

- Los sistemas de recarga deben ser estandarizados a nivel nacional.

\section{3) Marco regulatorio}

- Los incentivos por parte del gobierno son clave para la incursión de la tecnología en el país. Algunos tipos de incentivos expuestos son:

- Reducción del arancel e impuestos anuales

- Concesión de espacios públicos para sistemas de recarga

- Producción y ensamble nacional

- Recambio de tecnología - bonos

- Publicidad visual exterior

- Circulación - movilidad

- Las tecnologías relacionadas con los EV se deben incluir en el marco regulatorio nacional, como el RETIE

- Los incentivos deben estudiarse y aplicarse por nicho o sector para que garanticen dar solución a los requerimientos específicos.

\section{4) Factor Ambiental y Social}

- Las nuevas generaciones tienen mayor conciencia sobre las condiciones ambientales, cambio climático y eficiencia energética. Se identifican personas entre los 25 y los 34 años como usuarios potenciales de esta tecnología.

- La implementación de los EV puede reducir el impacto en salud pública por material particulado.

\section{5) Factores Económicos y Comercialización}

- Los costos de compra de los EV son altos y debe evaluarse la rentabilidad por costos de operación.

- De acuerdo con los precios internacionales y de importación de los EV, se estima que los usuarios potenciales que invertirían en este tipo de vehículo deben tener un poder adquisitivo alto (aproximadamente el 5\% de la población colombiana).

- Se debe desarrollar tecnología EV que se acople a las condiciones regionales.

Los planteamientos y conclusiones de las tres rondas Delphi se sintetizan por temas [13]. En la Fig. 11 se muestra el tipo de EV sugerido para cada uso de acuerdo con sus características. En la Fig. 12 se presenta el tipo de recarga y la infraestructura según las necesidades e inquietudes manifestadas por el tipo de usuario; en ésta se hace énfasis a la importancia de la intervención del gobierno para garantizar la infraestructura de acuerdo con la demanda. En la Fig. 13 se resalta claramente la falta de desarrollo en las tecnologías periféricas, principalmente de las baterías. En este caso particular, en la tercera ronda, se plantearon temas principales que deben ser trabajados por los grupos de investigación para que se propicie una maduración en la tecnología apropiada para los EV. Finalmente, en la Fig. 14 se presenta una síntesis sobre los impactos identificados desde varias áreas del saber alrededor de la implementación de los EV. 
Conclusiones Rondas Delphi sobre Implementación de EVs en Colombia

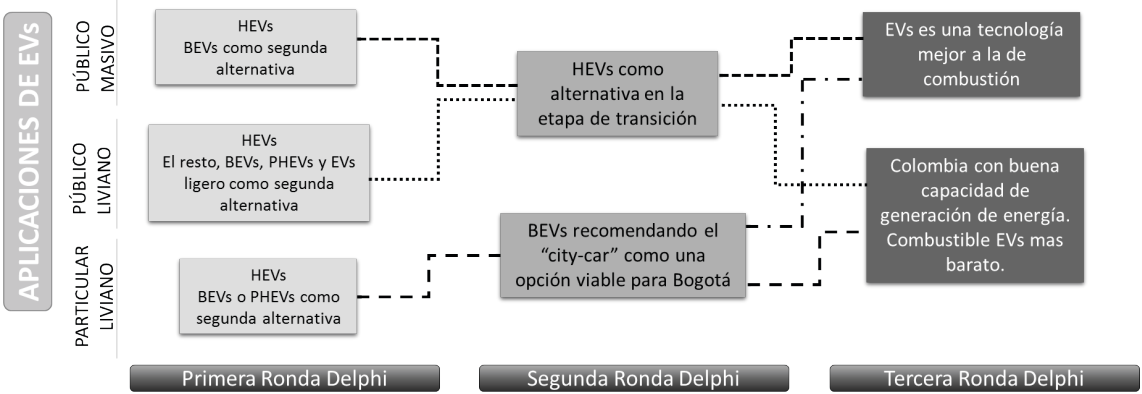

Fig. 11. Síntesis Resultados Rondas Delphi - Aplicaciones de los EV. Fuente: [13]

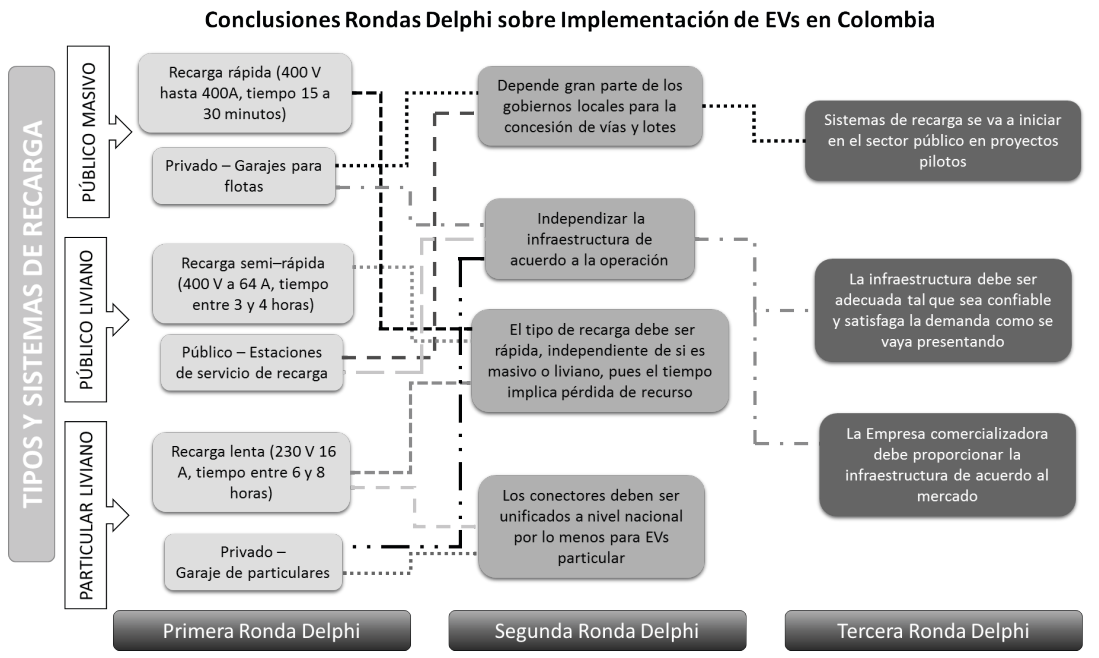

Fig. 12. Síntesis Resultados Rondas Delphi - Tipos y Sistemas de Recarga. Fuente: [13]

Conclusiones Rondas Delphi sobre Implementación de EVs en Colombia

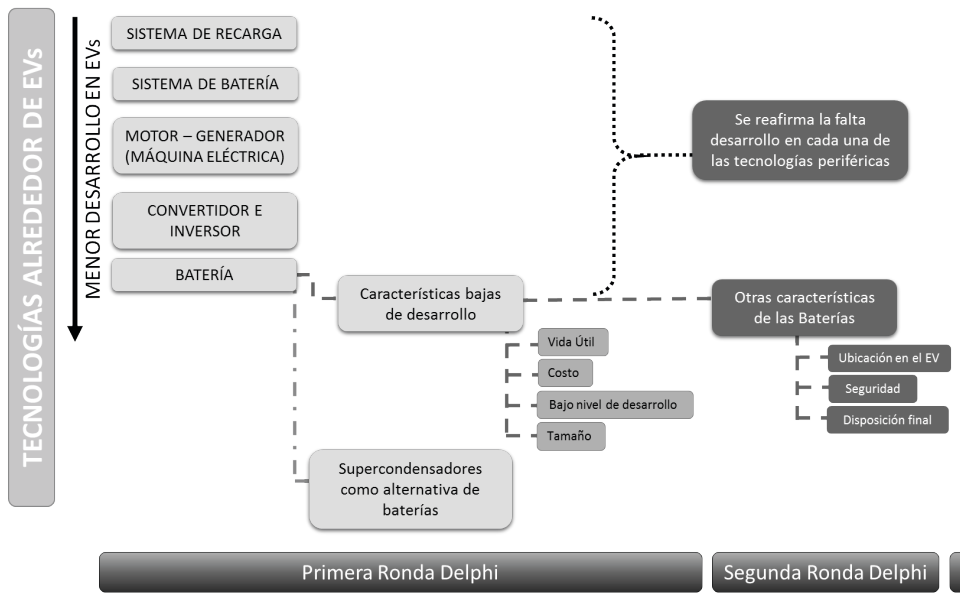

Fig. 13. Síntesis Resultados Rondas Delphi - Tecnologías Periféricas. Fuente: [13] 


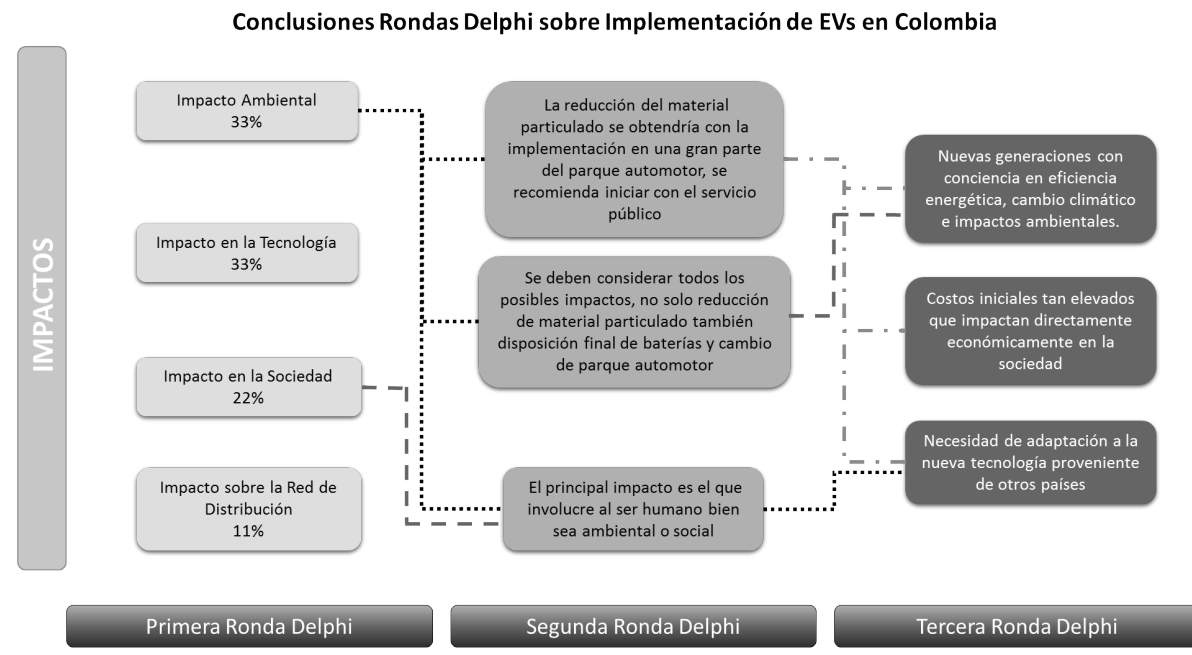

Fig. 14. Síntesis Conclusiones Rondas Delphi -- Impactos de la Implementación de los EV. Fuente: [13]

\section{Planteamiento De Escenarios}

Para la determinación de los escenarios se establecieron los siguientes parámetros:

- El horizonte de tiempo es de 17 años, es decir al año 2030. Este tiempo fue establecido posterior a una de la rondas Delphi.

- Los tipos de escenarios que se plantearon fueron normativos (optimistas, pesimistas) por la naturaleza del enfoque de la implementación.

- Las dos variables identificadas como de gran influencia sobre la implementación de los EV son: La existencia o no de incentivos y el desarrollo de la tecnología.

Estas dos variables serán los ejes para la definición de los escenarios [13], de esta manera, los cuatro cuadrantes estarán determinados de la siguiente forma:

a. Primer Escenario: Incentivos para la implementación con un buen desarrollo de las tecnologías relacionadas con los EV.

b. Segundo Escenario: Un buen desarrollo de las tecnologías alrededor de los EV, pero sin incentivos para su implementación.

c. Tercer Escenario: Incentivos para la implementación de los EV, pero sin un buen desarrollo de la tecnología relacionada con los EV.

d. Cuarto Escenario: Implementación sin un buen desarrollo tecnológico de los EV y sin incentivos por parte del gobierno.

Las características de los cuatro escenarios fueron obtenidas como resultados de los procesos de vigilancia y prospectiva tecnológica y se muestran en la Fig. 15. Estas características están en función de las dos variables identificadas, teniendo en cuenta cada factor descrito en la vigilancia tecnológica (sistemas de recarga, tecnologías como la batería, frenos regenerativos, etc.) [13].
Asimismo, se estiman factores como: el ambiental y el social que no son estudiados estadísticamente como los otros planteados.

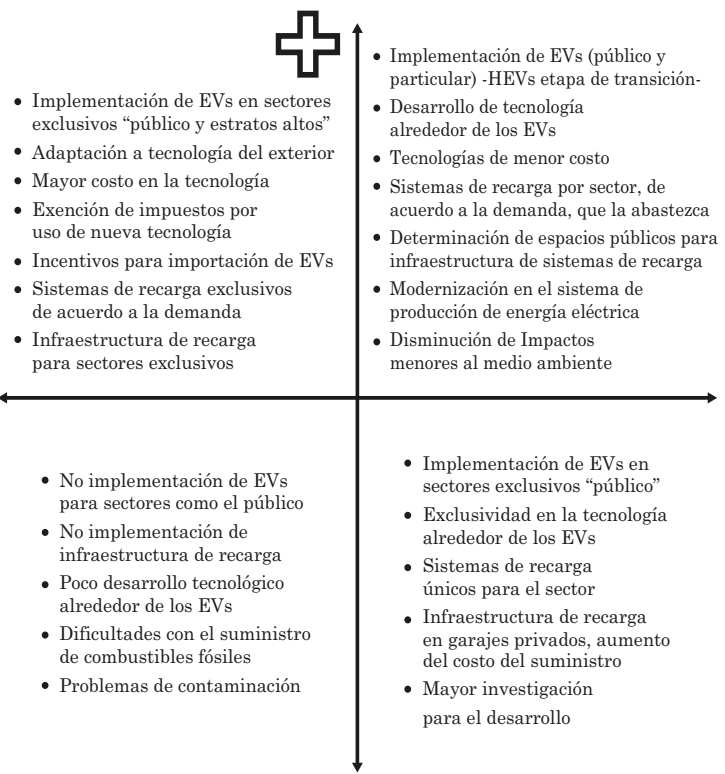

Fig. 15. Esquema de Escenarios Planteados. Fuente: [13]

Los desarrollos tecnológicos alrededor de los EV, desde el ámbito investigativo y de propiedad intelectual, están dirigidos principalmente a las baterías y a la infraestructura de recarga.

Los temas con mayor cantidad de publicaciones científicas son, por un lado, los supercondensadores, clasificados dentro de los sistemas de almacenamiento de energía; y por otro, los convertidores e inversores, clasificados dentro de los temas de infraestructura de recarga. 
El interés de investigación sobre los EV para uso masivo prima sobre los EV para uso particular, sin embargo, las primeras tecnologías asociadas son desarrolladas y comercializadas para uso particular.

En este ejercicio se pone en evidencia la falta de relación universidad-empresa. Tanto los registros de patentes como los de artículos científicos demuestran que aún no existe esa relación que permita a las empresas apoyarse en las universidades para el desarrollo de nuevas tecnologías, y a su vez, a las universidades apoyarse en las empresas para patrocinio y enfoque de investigación en temas de mercado.

\section{Conclusiones}

Como resultado del desarrollo de los procesos metodológicos implementados, se identificaron las características relevantes de la incursión de los EV en Colombia. Se considera que el bajo desarrollo de las tecnologías, principalmente de las baterías, puede ser un gran obstáculo para su masificación.

De las inquietudes planteadas durante las rondas Delphi se derivan propuestas para desarrollar estudios alrededor de los impactos sociales y así determinar si los colombianos estamos preparados o no para asimilar el ingreso de la tecnología. Lo anterior se debe a que Colombia no es un país desarrollador de tecnología sino que es un país consumidor.

Las redes inteligentes y el consumo de energía son dos temas de interés que se relacionan directamente con la implementación de los EV. El primero incluye a los EV dentro de su esquema de operación mientras que el consumo de energía lo asocia al uso racional de energía y disminución de gases para evitar los gases de efecto invernadero.

Para hacer viable de la implementación de los EV en Colombia se depende altamente de los incentivos y de las regulaciones que se definan. Este marco regulatorio debe ser liderado y controlado por una entidad gubernamental tal que permita el fortalecimiento del desarrollo tecnológico y la correcta penetración de la tecnología.

Los impactos social, ambiental, económico, tecnológico y sobre la red de distribución tienen la misma proporción en importancia dentro del estudio. Sin embargo, para la etapa de transición de la penetración de los EV debe seleccionarse la tecnología adecuada de manera que los impactos no generen un estancamiento del desarrollo tecnológico. Asimismo, debe trabajarse en la necesidad de adaptación de la tecnología proveniente de otros países, por la naturaleza de ser un país consumidor más que productor y desarrollador.
La infraestructura de recarga debe contar con conectores unificados a nivel nacional de forma que pueda existir la traslación de vehículos intermunicipalmente. De igual manera, la infraestructura debe ser confiable, rápida y, sobre todo, debe satisfacer la demanda de acuerdo con la tendencia de penetración.

\section{REFERENCIAS}

[1] Ping Guo and Peng Liu, "Research on development of electric vehicles in China," in 2010 International Conference on Future Information Technology and Management Engineering, 2010, vol. 1, pp. 94-96. DOI: 10.1109/ FITME.2010.5655778

[2] J. Pérez Turpin and I. Blasco Mira, Metodologías de Investigación en las Ciencias de la Actividad Física y el Deporte: Ampliando Horizontes. Alicante: ECU, p. 309, 2007.

[3] C. I. Camargo Bareño, "Transferencia tecnológica y de conocimientos en el diseño de sistemas embebidos," M.S thesis, Dept. Elect. and Electron. Eng. Univ. Nacional de Colombia, Bogotá, Colombia, 2011.

[4] E. Astigarraga, "El Método Delphi," Universidad de Deusto, Mundaiz, 2006.

[5] F. Tobar, "Análisis de Tendencias y Construcción de Escenarios," Argentina, 2006.

[6] M. L. Rodríguez, "Metodologías de la Investigación," 2010. [En línea]. Disponible en: https://metodologiasdelainvestigacion.wordpress.com/2010/12/10/la-tecnicaprospectiva-de-los-escenarios/. [Accessed: 15-Oct-2013].

[7] P. Morcillo, "Vigilancia e inteligencia competitiva: fundamentos e implicaciones," Rev. Investig. en Gestión la Innovación y Tecnol., vol. 17, pp. 10-17, 2003.

[8] M. Delgado-Fernández, J. L. Pino Mejías, F. M. Solís Cabrera, and R. del C. Barea Barrera, "Evaluación integrada de la innovación, la tecnología y las competencias en la empresa," Rev. Investig. en Gestión la Innovación y Tecnol., vol. 47, p. 14, 2008.

[9] A. M. Gomes de Castro, "Estúdios de futuro: fundamentos, metodologias, técnicas," Taller sobre Prospectiva de Cadenas Productivas-Colciencias, 2005. [En línea]. Disponible en: https:/campusvirtual.univalle.edu.co/ moodle/pluginfile.php/313404/mod_resource/content/0/ fuerzas_propulsoras_restrictivas.pdf..

[10] B. Larsen, "Final report: Colombia: Cost of environmental damage: A socio-economic and environmental health risk assessment," Bogota, 2004.

[11] M. Delgado-Fernández, M. Infante-Abreu, Y. Abreu-Lee, O. Infante-Pérez, A. Díaz-Batista, And J. Martínez-Moreno, "Vigilancia Tecnológica En Una Universidad De Ciencias Técnicas / Technological Surveillance In A Technical Sciences University," Ingeniería Industrial, Vol. 32, No. 1. Pp. 69-75, 03-Jul-2011.

[12] K. Al-Mabrouk and J. Soar, "Building a framework for understanding and improving information technology transfer process in the Arab countries," Proceedings of the 9th IBIMA Conference: Information Management in Modern Organisations - Trends \& Challenges. International Business Information Management Association, 01-Jan-2008.

[13] M. V. Díaz Merchán, "Vigilancia tecnológica y prospectiva del vehículo eléctrico y tecnologías periféricas,” M.S thesis, Dept. Elect. and Electron. Eng., Univ. Nacional de Colombia, Bogota, Colombia, 2013. 